\title{
Calculation and Data Processing Method for Aircraft Maneuvering Performance
}

\author{
Song Limin ${ }^{\text {a }}$, Liu Xiujuan ${ }^{\mathrm{b}}$, Cao Wenbin ${ }^{\mathrm{c}}$, Li Song ${ }^{\mathrm{d}}$ and Yuan Lei ${ }^{\mathrm{e}}$ \\ Aviation University of Air Force, Changchun, Jilin, China \\ aliminsong_2001@163.com, ${ }^{b} x i u j u a n l i u @ 126 . c o m, ~{ }^{c} \mathrm{Cao}$ \\ Wenbin@126.com, ${ }^{d}$ lisong4885@126.com, ${ }^{e}$ Yuanlei@163.com
}

Keywords: flight performance; calculation; data processing

\begin{abstract}
The quality of flight performance directly determines the execution of flight tasks. For the establishment of a simple and effective methods and calculation of maneuver performance, a lot of work were completed. By the principle, method and steps of calculation maneuvering the aircraft flight performance and MATLAB software, relevant personnel can know related parameters' influence on flight performance as intuitive and quantitative. There provides certain reference for flight.
\end{abstract}

\section{Introduction}

Aircraft flight performance refers to the ability of the aircraft to be used as a regular or unsteady motion along the flight path, including basic flight performance, maneuver flight performance, take-off and landing performance, and cruise performance [1]. The flight performance is directly related to the performance of aircraft technical and tactical performance and flight safety assurance. While maneuvering flight is fighter technology basic flight, flight training is essential in training courses to improve the quality of flight training and ensure flight safety, workload and error are large, but not refined processing will come some unnecessary trouble [2]. Therefore, in order to determine the flight performance characteristics, the preparation of motor performance computing program is very necessary [3] [4].

\section{Equation of Motion}

The control law of maneuvering flight calculation adopts the variation law of the load with the track angle. The equations of motion of the aircraft in the flight path coordinate system or the air current coordinate system are:

$$
\begin{aligned}
& m\left(\frac{d V_{x}}{d t}+V_{z} \omega_{y}-V_{y} \omega_{z}\right)=F_{x} \\
& m\left(\frac{d V_{y}}{d t}+V_{x} \omega_{z}-V_{z} \omega_{x}\right)=F_{y} \\
& m\left(\frac{d V_{z}}{d t}+V_{y} \omega_{x}-V_{x} \omega_{y}\right)=F_{z}
\end{aligned}
$$

Plane Maneuver Flight. There are many kinds of maneuvering flight, which is circling maneuver in the horizontal plane, is the loop maneuver in a vertical plane, and oblique loop is in oblique plane maneuver is completed, a part of subduction and can be seen as the track loop jump [5]. As a simplification, it can be seen as a planar maneuver. The kinetic equation of the particle can be transformed into:

$$
\begin{gathered}
\frac{g}{G} \cdot \frac{d V}{d t}=P \cos \left(\alpha+\varphi_{p}\right)-Q-G \cos \lambda \sin \theta \\
V \cdot \frac{g}{G} \cdot \frac{d \theta}{d t}=\left[Y+P \cos \left(\alpha+\varphi_{p}\right)\right] \sin \delta-G \cos \lambda \cos \theta
\end{gathered}
$$

Among $\delta=90^{\circ}, \gamma=0^{\circ}, \phi=0^{\circ}, z=0$. The plane loop flight, dive, slimbing's curve is the same as the equation of motion of a loop. And line segment at that time, $d \theta / d t=0^{\circ}$ and 
when $\delta=\gamma, \theta=\phi=0^{\circ}, y=0$, the plane was circling.

Upward Turn. The rising turn of the aircraft is not in the same plane, so it is necessary to establish the equation of motion:

$$
\begin{aligned}
& \frac{d V}{d t}=\frac{g}{G}\left[P \cos \left(\alpha+\varphi_{p}\right)-Q-G \sin \theta\right] \\
& \frac{d \theta}{d t}=\frac{g}{V}\left(n_{y} \cos \gamma_{s}-\cos \theta\right) \\
& \frac{d \psi_{s}}{d t}=-\frac{g}{V} \cdot \frac{n_{y}}{\cos \theta} \sin \gamma_{s} \\
& \frac{d L}{d t}=V \cos \theta \cos \psi_{s} \\
& \frac{d H}{d t}=V \sin \theta
\end{aligned}
$$

Up turn requires input heading angle, overload, aircraft slope, engine condition.

Calculation Flow Chart. The flow chart is as follows:

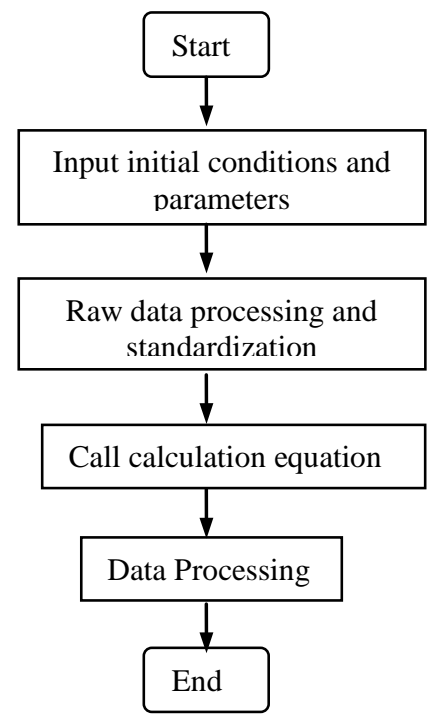

Fig. 1 The flow chart

\section{Data Processing}

Original Data Selection. An important task of aircraft performance calculation is to obtain and deal with the original data. The original data can be used to reduce the burden of data processing, but the data obtained through technical specification is limited [6] [7]. In order to make the calculation result closer to the accurate result, we must strengthen the data calculation method, and determine which kind of interpolation method is used according to the changing trend of the data curve. This is mainly used for linear interpolation (see formula 4) and three point parabolic interpolation (see formula 5).

$$
\begin{gathered}
y(x)=\frac{\left(x-x_{2}\right)}{\left(x_{1}-x_{2}\right)} f\left(x_{1}\right)+\frac{\left(x-x_{1}\right)}{\left(x_{2}-x_{1}\right)} f\left(x_{2}\right) \\
y(x)=\frac{\left(x-x_{2}\right)\left(x-x_{3}\right)}{\left(x_{1}-x_{2}\right)\left(x_{1}-x_{2}\right)} f\left(x_{1}\right)+\frac{\left(x-x_{1}\right)\left(x-x_{3}\right)}{\left(x_{2}-x_{1}\right)\left(x_{2}-x_{3}\right)} f\left(x_{2}\right)+\frac{\left(x-x_{1}\right)\left(x-x_{2}\right)}{\left(x_{3}-x_{1}\right)\left(x_{3}-x_{2}\right)} f\left(x_{3}\right)
\end{gathered}
$$

Data Processing Methods. For loop, hover, dive, jump, oblique loop, flip, wingover, inverted bucket half turn to increase input phase overload and thrust state, in order to accelerate the speed of operation, put these data into a data file, an input. Because the equation of motion is simple, the calculation model is not suitable for the calculation, so it is not suitable for the calculation [8].

In this study, matlab combined with $\mathrm{C}$ language as an analytical tool, the whole algorithm is divided into test data preprocessing module, data calculation module and the results of the 
processing module. After a preliminary test, some results are shown in figure 1:

\section{Calculation Results and Improvement}

Based on the analysis of the mechanical theory as the foundation, designed the soccer robot pick the ball institutions optimal design process, found aim function, select design variables and the corresponding optimization. The test results is shown as Figure. 2.

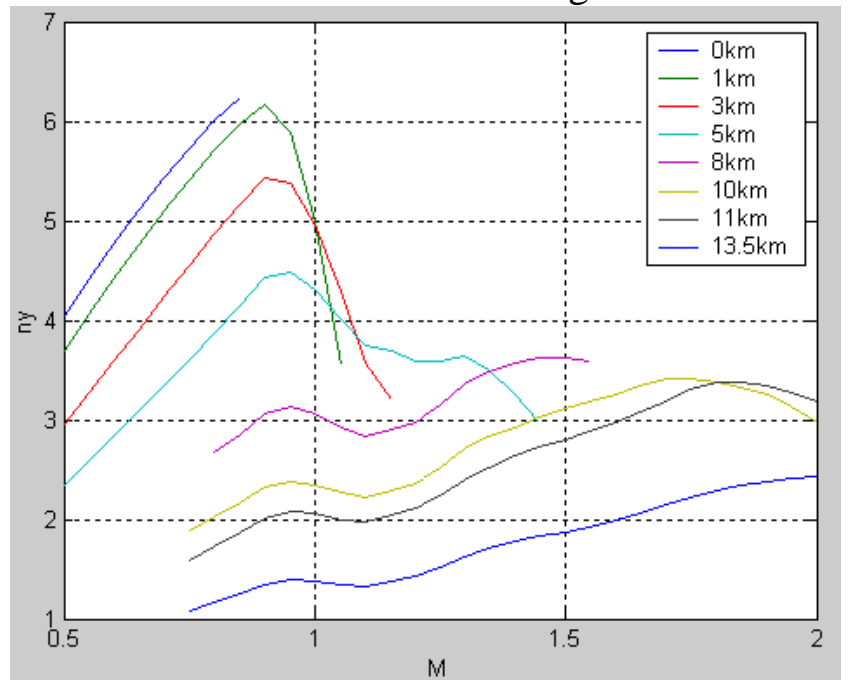

(a)

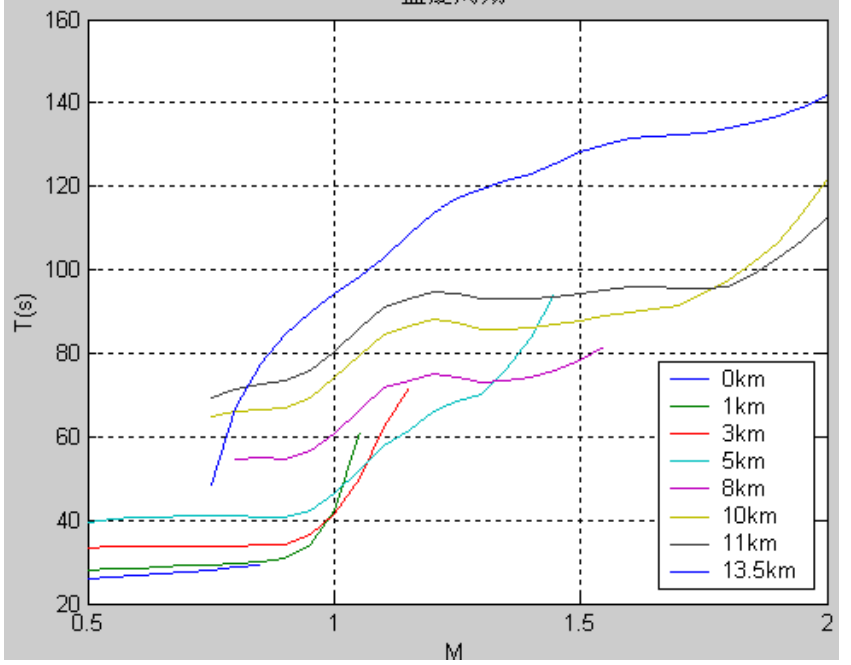

(b)

Fig. 2 Test results

Examples show that compared with the simple estimation method, the calculation program has better precision, less sample data, the relationship can be a very good response to the flight performance parameters, and can draw the related curve, make the results more intuitive and save a lot of time. In this paper, the research process is only a preliminary attempt, the next step will be the results of the data fitting and regression equation to do further research.

\section{References}

[1] Xuan-ping Zhang, Wei-hu Yu, Jing-jing Liang, Bo Liu School of Electronics and Information Engineering, Xi'an Jiaotong University, Xi'an 710049, Shaanxi, People's Republic of China. Entropy Regularization Method for Coordinated Multiple Target Assignment[A]. Proceedings of 2010 3rd IEEE International Conference on Computer Science and Information Technology VOL.5[C]. 2010

[2] Ronald A. Hess. Modeling Pilot Detection of Time-Varying Aircraft Dynamics. Journal of 
Aircraft . 2012

[3] Dogan, Atilla, Kaewchay, Komkrit. Probabilistic human pilot approach: Application to microburst escape maneuver. Journal of Guidance Control and Dynamics . 2007

[4] Hess, Ronald A., Marchesi, Federico. Analytical assessment of flight simulator fidelity using pilot models. Journal of Guidance Control and Dynamics . 2009

[5] Bombardier Aerospace Group North America. Bombardier Selects PACE as Development Partner for Computerized Airplane Flight Manual. . 2005

[6] Gao, Tong, Lu, Xi-Yun. Insect normal hovering flight in ground effect. The Physics of Fluids . 2008

[7] Tien Truong, Jihoon Kim, Min Jun Kim, Hoon Cheol Park, Kwang Joon Yoon, Doyoung Byun. Flow structures around a flapping wing considering ground effect[J]. Experiments in Fluids . 2013 (7)

[8] J.Su, J.H.Tang, C.H.Wang, et al. A numerical investigation on the ground effect of a flapping flying bird. The Physics of Fluids . 2013 